\title{
Cardiac vagal control and theoretical models of co-occurring depression and anxiety: A cross-sectional psychophysiological study of community elderly
}

Hsi-Chung Chen ${ }^{1,2}$, Cheryl CH Yang ${ }^{3}$, Terry BJ Kuo ${ }^{3}$, Tung-Ping Su ${ }^{4,5}$ and Pesus Chou ${ }^{2^{*}}$

\begin{abstract}
Background: In order to elucidate the complex relationship between co-occurring depression and anxiety with cardiac autonomic function in the elderly, this study examined the correlation between cardiac vagal control (CVC) and pre-defined, theoretical factors from the Hospital Anxiety and Depression Scale (HADS).

Methods: Three hundred fifty-four randomly selected Chinese male subjects aged $\geq 65$ years and living in the community were enrolled. CVC was measured using a frequency-domain index of heart rate variability.

Results: Confirmatory factor analysis showed that the flat tripartite model of HADS provided a modest advantage in model fit when compared with other theoretical factor solutions. In the flat tripartite model, there was a significant negative association between anhedonic depression and CVC. In contrast, autonomic anxiety showed a significant positive correlation with CVC. In the hierarchical tripartite model, negative affectivity was not directly associated with CVC; instead, it had positive and negative indirect effects on CVC via autonomic anxiety and anhedonic depression, respectively. As scores for negative affectivity increased, these specific indirect effects diminished.

Conclusions: Among competing models of co-occurring depression and anxiety, constructs from tripartite models demonstrate fair conformity with the data but unique and distinct correlations with CVC. Negative affectivity may determine the relationship of anhedonic depression and autonomic anxiety with CVC. Separating affective symptoms under the constructs of the tripartite models helps disentangle complex associations between co-occurring depression and anxiety with CVC.
\end{abstract}

Keywords: Cardiac vagal control, Co-occurring depression and anxiety, Heart rate variability, The Hospital Anxiety and Depression Scale, Tripartite model

\section{Background}

It is well known that depression influences the development of cardiovascular disease in several ways [1-4]. However, several features characterize the association between depression and cardiovascular events. First, there are gender differences in cardiovascular disease and comorbid depression [5]. Second, the differential

\footnotetext{
* Correspondence: pschou@ym.edu.tw

${ }^{2}$ Community Medicine Research Center \& Institute of Public Health, National Yang-Ming University, Shih-Pai, Taipei 112, Taiwan

Full list of author information is available at the end of the article
}

magnitude of association has prompted researchers to identify high-risk groups with clusters of cardiac-noxious depressive symptoms [6-9]. Third, anxiety symptoms, which commonly co-occurred with depression, have also been linked to adverse cardiac outcomes and are thought to significantly confound the effect of depression on cardiovascular disease [10-12]. In order to further elucidate the nature of gender differences, heterogeneous relationships, and confounding anxiety symptoms in the association between depression and cardiovascular diseases, it 
is important to explore the role and relevance of biological substrates that underlie theses patterns.

Cardiac vagal control (CVC) reflects the extent to which the tonic vagal activity influences the heart [13]. High resting CVC and a high capacity for withdrawing CVC during environmental demand are thought to facilitate physical and psychological function. In contrast, low resting $\mathrm{CVC}$ and a low capacity for withdrawing $\mathrm{CVC}$ are thought to predict poor outcomes. Pathways by which the frontal cortex modulates vagal activity via subcortical inputs have been identified [14]. Therefore, CVC exerts regulatory control over attentional and emotional systems as well as behavioral flexibility $[15,16]$.

Impaired $\mathrm{CVC}$ has been suggested to mediate the link between depression and cardiovascular events $[17,18]$. In parallel, there are also gender differences $[19,20]$ and heterogeneous relationships [21-23] in the associations between depressive symptoms and CVC. Moreover, because depression and anxiety are broad, interrelated, affective constructs, co-occurring anxiety symptoms may confound or exacerbate impaired CVC as well $[18,24]$. These factors obscure the clinical utility of treating affective symptoms for the prevention and treatment of cardiovascular disease. Therefore, we need genderspecific studies that concurrently deal with the associations between co-occurring affective symptoms and heterogeneous patterns with CVC. As such, pre-defined, theory-based management of psychopathology is crucial for preventing post-hoc arbitrary manipulation of predictive variables [24].

Early research on the structure of depression and anxiety resulted in the two-factor model that emphasized two orthogonal dimensions: negative affect and positive affect. Negative affect is a shared, non-specific component to both depression and anxiety, whereas low positive affectivity is a specific factor primarily related to depression [25]. Clark and Watson (1991) extended the two-factor model to the tripartite model, which includes anxious arousal [26]. In the tripartite model, negative affectivity is a shared characteristic between depression and anxiety. The measure of negative affectivity is strongly related to the trait of neuroticism $[27,28]$. Negative affectivity can be regarded as the distinctive core emotional process of neuroticism [29]. Hence, it can be expressed as not only worry, nervousness, and tension, but also other negative emotional states, such as guilt, anger, and self-dissatisfaction [30]. Investigators further modified the flat tripartite model to a hierarchical structure in order to explain the co-morbidity and specificity of anxiety and depression at the level of both symptoms and disorders [31,32]. The hierarchical tripartite model posits a higher order negative affect factor and two lower order factors specific to the unique components of depression and anxiety (low positive affect and anxious arousal, respectively) in order to describe the relationship among measures of negative affect $[26,33]$. In the hierarchical arrangement of the three-factor model, a second-order dimension of negativity was conceptually extracted from corresponding lower order depression and anxiety. Consequently, the traditional syndromes of depression and anxiety represent narrow constructs that are highly interrelated. In contrast, the negative affectivity dimension emerges as a broader, more general construct that represents the strong degree of overlap between the lower order syndromes [34]. This model also takes into account the overlap among neuroticism, anxiety, and depression and is applicable to both clinical and non-clinical samples [35]. Multi-group analyses have suggested that the model can be effectively applied to older populations [36].

The above-mentioned series of a-priori defined models (i.e. the two-factor model and flat and hierarchical tripartite models) for co-occurring depression and anxiety have been used to examine the psychometric properties of paper-and-pencil measurements [37-39]. These models simultaneously handle the issues of symptomatic heterogeneity and co-occurring affective symptoms; therefore, with distinct affective constructs, they are also good approaches for tackling the complex relationship between affective symptoms and innate biological factors. In the two-factor model, positive affectivity rather than negative affectivity is strongly influenced by endogenous rhythms [40]. In the tripartite model, low positive affectivity is related to reduced right-hemisphere activity, and high negative affectivity is associated with increased left-hemisphere activity. Specifically, anxious arousal was related to increased right-hemisphere activity [41,42]. In addition to brain activity, the tripartite model has also been used to explore association patterns between individual factors and serum cortisol levels [42,43]. Because factors derived from the aforementioned theoretical-based models activate different brain areas, and chronotropic control of heart rate is right-hemisphere dominant, it is possible that different affective constructs correlate distinctively with CVC. However, this potential unique relationship between affective constructs and CVC has never been examined.

Thus, the present study aimed to disentangle the correlation patterns between co-occurring depression and anxiety with CVC in a gender-specific sample. The conformity of competing theory-based models for cooccurring depression and anxiety in an elderly Chinese male population was examined first. Furthermore, each theoretical model was applied to test and compare their unique correlation with CVC. It is hypothesized that each theory-derived construct behaves differently in terms of their relationship with CVC. 


\section{Method}

Participants

This study was a part of the community health survey conducted by the Community Medicine Research Center at the National Yang-Ming University in Taiwan. The subject sampling method has been reported previously [19]. Briefly, in 2007, 392 elderly men (65 years of age and older) dwelling in an urban community located in northern Taipei were randomly selected to participate in the study. Demographic data and a self-reported medical history with respect to hypertension, diabetes, cardiovascular disease, exercise habits, and substance exposure were collected. Exclusion criteria included: (1) failure to provide a history of medical illnesses, (2) not receiving regular treatment for medical illnesses, (3) subjects with conditions which affected cardiovascular fluctuations (i.e. frequent atrial fibrillation or ventricular contractions), and (4) subjects taking antidepressants. A total of 38 potential subjects were excluded, and data from 354 eligible subjects were analyzed. All participants provided written informed consent, and the Ethics Committee of National Yang-Ming University approved this study.

\section{Measurement of depressive and anxiety symptoms}

The Hospital Anxiety and Depression Scale (HADS) is a valid instrument for screening or measuring both clinical and sub-clinical depression and anxiety in the general population [44]. During its development, the authors excluded symptoms that might arise from somatic aspects of illness, which made it useful in the elderly population. The authenticity of the Mandarin version of HADS has been previously investigated [45].

Given the aforementioned evolution of the phenotypic structures of depression and anxiety, HADS had been reported with respect to several theoretical factor solution models. The item distributions of the unitary factor model of Razavi (1990) [46], the original two-factor model of Zigmond and Snaith (1983) [47], and the tripartite three-factor model (flat and hierarchical) of Clark and Watson (1991) [26] are shown in Figure 1. Dunbar et al.. (2000) [37] later split the original anxiety subscale of HADS into two factors: negative affectivity and autonomic anxiety. All items on the depression subscale were regarded as good markers for anhedonic depression (Figure 1).

\section{Measurements of cardiac vagal control}

After a 10-min rest in a seated position, a lead-I electrocardigram (ECG) was taken for $5 \mathrm{~min}$. A heart rate variability (HRV) analyzer (SSIC, Enjoy Research Inc., Taiwan) acquired, stored, and processed the ECG signals. Signals were recorded using an 8-bit analog-to-digital converter with a sampling rate of $512 \mathrm{~Hz}$. All peaks of the digitized ECG signals were detected using a spike detection algorithms [48]. The mean and standard deviation of the amplitude and duration of all spikes were used as the standard QRS template. According to the likelihood in fitting the standard QRS template, ventricular premature complex and noise were excluded, and only valid QRS complexes were collected in the procedure of $\mathrm{R}-\mathrm{R}$ interval rejection. All $\mathrm{R}-\mathrm{R}$ intervals were used to derive a temporary mean and standard deviation for standard reference. Each R-R interval was then examined. If the standard score of an $R-R$ value exceeded 3, it was regarded as erroneous or nonstationary and was then rejected. Stationary R-R interval values were resampled and interpolated at a rate of $7.11 \mathrm{~Hz}$ to produce continuity in the time domain. A nonparametric method of fast Fourier transformation was utilized to perform power spectral analysis. The direct current component was deleted, and a Hamming window was used to attenuate the leakage effect. The power spectrum of high frequency (HF: 0.15-0.40 Hz) was defined as the measurement of CVC. The spectral power of HF was logarithmically transformed to correct the skewed distribution.

\section{Data analysis}

The Statistical Package for the Social Science (SPSS) version 8.0 and the Linear Structural Relations (LISREL) version 8.2 were used to analyze all data. The factor structure of HADS was determined using Confirmatory factor analysis (CFA) with the SIMPLIS language in the LISREL system. The weighted least-square estimator was used to evaluate model fit as this method of estimation can be used reliably with ordered categorical level data and dependably with modest samples sizes. No model revision was made for poorly fitting solutions. In the hierarchical tripartite model, the covariance of firstorder factors of anhedonic depression and autonomic anxiety was constrained to be zero.

Multiple goodness-of-fit tests were used to evaluate the models. A root mean square error of approximation (RMSEA) with values $<0.08$ indicated a good fit, while values $>0.10$ suggested an unsatisfactory fit. Comparative Fit Index $(\mathrm{CFI})>0.90$ indicated a good fit to the data, while the Consistent Akaike information criterion (CAIC), Akaike information criterion (AIC), and expected cross validation index (ECVI) allowed for comparison between models [37]. These parameters comprise the three major categories of goodness-of-fit tests: absolute index (RMSEA, ECV), incremental index (CFI), and parsimony index (AIA, CAIC). Thus, this combination examines model fitness more comprehensively.

In multivariate analyses, multiple linear regressions were performed to analyze the relationship between factor solutions of HADS and CVC. Age, education, body mass index, smoking status, weekly frequency of exercise, 


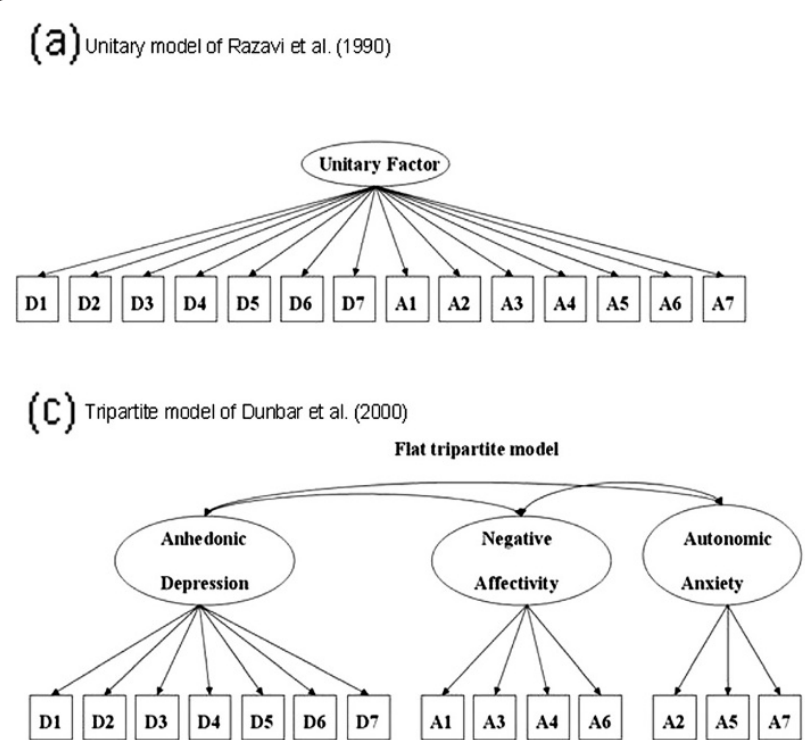

Depression Subscale of the HADS

D1 : Still enjoy things

D2 : Laugh and see funny side

D3 : Feel cheerful

D4 : Feel slowed down

D5 : Lost interest in appearance

D6 : Look forward with enjoyment

D7 : Enjoy book, radio, or TV (b) Two-factor model of Zigmond and Snaith (1983)
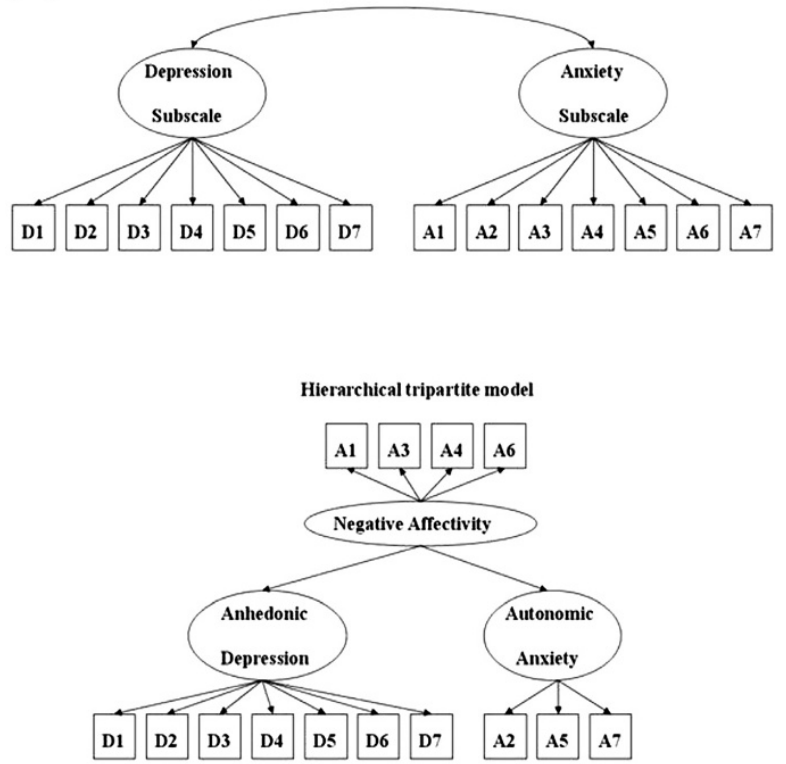

Anxiety Subscale of the HADS

A1 : Tense and wound up

A2 : Something awful about to happen

A3 : Worrying thoughts

A4 : Sit at ease and feel relaxed

A5: 'Butterflies' in stomach

A6: Feel restless

A7 : Sudden feelings of panic

Figure 1 Diagrammatic illustration of the factor structures of the Hospital Anxiety and Depression Scale.

sites of data collection, and history of hypertension, diabetes, and coronary heart disease were forced into the models to adjust for potential confounding effects. Because of the reported non-linear relationship between biological markers and depression $[19,42,43]$ and the relatively low symptom severity of depression in the present study, the depression subscale of HADS was transformed to ordinal scores based on the quartile distribution and the suggested cut-off point for depression subscale of HADS. The optimal cut-off point for the depression subscales of HADS in community-dwelling Chinese elderly is 6 [49]; therefore, the lowest two quartiles $(<5)$ were grouped as the first class, and the third quartile (5-6) and forth quartile $(\geq 7)$ were re-classified as 2 and 3 , respectively.

Compared with the flat tripartite model, the hierarchical arrangement offers additional information about how each construct influences CVC. The a-priori defined hierarchical tripartite model implied that the higher order construct of negative affectivity might confer indirect effects on CVC by the following pathway: negative affectivity $\rightarrow$ anhedonic depression $\rightarrow$ CVC and negative affectivity $\rightarrow$ autonomic anxiety $\rightarrow$ CVC. In the mediation models, the total effect of negative affectivity on CVC can be further partitioned into a direct effect and two specific indirect effects. A direct effect is interpreted as the effect of negative affectivity on CVC that is independent of the pathway through intervening variables (i.e. anhedonic depression and autonomic anxiety). The indirect effect is the amount by which two cases who differ by one unit on negative affectivity are expected to differ in CVC through negative affectivity's effect on anhedonic depression or autonomic anxiety, which in turn affects CVC [50]. Indirect effects were statistically examined with the SPSS macro provided by Preacher and Haynes (2008) for assessing indirect effects in multiple mediator models [51]. This macro utilizes a bootstrapping strategy to test the validity of indirect effects. Several modern approaches are used to inference mediation effect. The conventional approach of Baron and Kenny infers the existence rather than quantifies the mediation effect [52]. Another popular inferential technique is the product of coefficients approach (i.e. Sobel test) [53,54]. The ratio of the path coefficients to its standard error is the test statistic for examining the null hypothesis with the $p$-value derived from the standard normal distribution. However, the Sobel test requires not only that the paths that constitute the indirect 
effects follow a multivariate normal distribution, but also that the sampling distribution of the total and specific indirect effects are normal [51]. In contrast, the bootstrapping strategy quantifies the indirect effect and makes no assumption of multivariate normal distribution in the sampling of indirect effects [50]. Simulation research also shows that bootstrapping is one of the more valid and powerful methods for testing the effects of intervening variables $[55,56]$. In short, current evidence suggests that the bootstrapping methods are superior to methods that assume symmetry or normality of the sampling distribution of the indirect effect. In the present study, the indirect effects of negative affectivity on CVC were bootstrapped with 5,000 samples; the bias-corrected and accelerated 95\% confidence interval (BCa 95\% CI) was estimated. Finally, since a higher order construct (i.e. negative affectivity) may moderate the effect of a lower level construct on CVC (i.e. anhedonic depression and autonomic depression), another SPSS macro provided by Preacher et al. (2007) was utilized to examine the conditional indirect effect (i.e. moderated mediation effect) [57].

\section{Results}

\section{Participants' characteristics}

Table 1 summarizes the demographic and clinical characteristics of participants. There were a total of 354 subjects (mean age: $77.9 \pm 5.2$ years), and $69.2 \%$ of the data was collected at the participants' homes. Older elderly individuals ( $\geq 75$ years of age) accounted for $77.9 \%$ of the subjects. The mean total score of HADS was 7.0 \pm 4.7 . The median HADS subscale scores were 4 for depressive symptoms (range: $0-17$ ) and 2 for anxiety symptoms (range: 0-14).

\section{The CFA for various factor solutions of HADS}

The factor models and accompanying fit indices are shown in Table 2. Among competing models, the unitary factor model of Razavi (1990) had the worst fit for the data. Except in RMSEA, the flat tripartite model of Dunbar et al. (2000) showed a modest advantage over the hierarchical tripartite model and the original two-factor model. In contrast, the two-factor model of Zigmond and Snaith (1983) provided a comparable fit to the hierarchical tripartite model across all model fit indices.

\section{Multiple linear regression analyses for the association between factor solutions and CVC}

Table 3 shows the relationship of various factor solutions of HADS with CVC. The bivariate correlation between depression subscores of HADS and CVC was examined first. The original depression subscores were not correlated with CVC (Pearson correlation coefficient: $r=-0.05, p=0.33)$. In contrast, the transformed ordinal
Table 1 Demographic and clinical characteristics of participants $(n=354)$

\begin{tabular}{|c|c|}
\hline & $(n, \%)$ \\
\hline \multicolumn{2}{|l|}{ Age (years) } \\
\hline $65-74$ & $78(22.0 \%)$ \\
\hline $75-79$ & $163(46.0 \%)$ \\
\hline$\geqq 80$ & $113(31.9 \%)$ \\
\hline \multicolumn{2}{|l|}{ Education (years) } \\
\hline$<7$ & $100(28.2 \%)$ \\
\hline $7-9$ & $69(19.5 \%)$ \\
\hline $10-12$ & $80(22.6 \%)$ \\
\hline$\geqq 13$ & $105(29.7 \%)$ \\
\hline \multicolumn{2}{|l|}{ Body mass index $\left(\mathrm{kg} / \mathrm{m}^{2}\right)$} \\
\hline$\geqq 25$ & $139(39.3 \%)$ \\
\hline \multicolumn{2}{|l|}{ Weekly frequency of exercise } \\
\hline$\geqq 1 /$ week & $292(82.5 \%)$ \\
\hline \multicolumn{2}{|l|}{ Smoking status } \\
\hline Current smoker & $47(13.3 \%)$ \\
\hline \multicolumn{2}{|l|}{ Sites of data collection } \\
\hline Home & $245(69.2 \%)$ \\
\hline Hospital & $109(30.8 \%)$ \\
\hline \multicolumn{2}{|l|}{ Medical history } \\
\hline Diabetes mellitus (n, \%) & $75(21.2 \%)$ \\
\hline Hypertension (n, \%) & $217(61.3 \%)$ \\
\hline Coronary heart disease (n, \%) & $141(39.8 \%)$ \\
\hline \multicolumn{2}{|c|}{ Hospital Anxiety and Depression Scale } \\
\hline Total score (mean \pm SD) & $7.0 \pm 4.7$ \\
\hline Depression subscore (mean \pm SD) & $4.8 \pm 3.2$ \\
\hline Quartile $1(0-2)$ & $78(22.0 \%)$ \\
\hline Quartile 2 (3-4) & $131(37.0 \%)$ \\
\hline Quartile 3 (5-6) & $60(16.9 \%)$ \\
\hline Quartile $4(\geqq 7)$ & $85(24.0 \%)$ \\
\hline Anxiety subscore (mean \pm SD) & $2.2 \pm 2.1$ \\
\hline Quartile 1 (0) & $87(24.6 \%)$ \\
\hline Quartile 2 (1) & $74(20.9 \%)$ \\
\hline Quartile 3 (2-3) & $113(31.9 \%)$ \\
\hline Quartile 4 ( $\geqq 4)$ & $80(22.6 \%)$ \\
\hline
\end{tabular}

subscale of depression showed a significant negative correlation with CVC (Pearson correlation coefficient: $r=-0.15, p=0.01)$. Therefore, the rescaled depression subscale was adopted in models II to IV. The factor derived from the unitary factor model of Razavi (1991) (model I) did not correlate with CVC. Throughout models II to IV, anhedonic depression (rescaled depression subscale) invariably showed a negative association with CVC. In model III, when the tripartite factors of Dunbar et al. (2000) were entered into the model, negative 
Table 2 Factor structure of the Hospital Anxiety and Depression Scale as determined by testing the fit of models derived from factor analysis

\begin{tabular}{|c|c|c|c|c|c|c|}
\hline Models & WLS $X^{2}$ (d.f.) & RMSEA & CFI & CAIC & AIC & ECVI \\
\hline Unitary factor model of Razavi et al.(1990) & $369.37(77)$ & 0.10 & 0.86 & 561.71 & 425.37 & 1.21 \\
\hline Two-factor model of Zigmond and Snaith (1983) & $297.08(76)$ & 0.09 & 0.89 & 496.29 & 355.08 & 1.00 \\
\hline \multicolumn{7}{|l|}{ Tripartite model of Dunbar et al.(2000) } \\
\hline Flat tripartite model & $283.05(74)$ & 0.09 & 0.90 & 496.00 & 345.05 & 0.98 \\
\hline Hierarchical tripartite model & $295.68(75)$ & 0.09 & 0.89 & 501.76 & 355.68 & 1.01 \\
\hline
\end{tabular}

Bold indicates best model fit as a function of model fit index criteria. Abbreviations: Weighted least-square (WLS), Root mean squared error of approximation $(R M S E A)$, Comparative fit index(CFI), Consistent Akaike information criterion (CAIC), Akaike information criterion ( $A / C)$ and expected cross validation index (ECVI).

affectivity had no direct effect on CVC $[\beta(\mathrm{SE})=-0.09$ (0.05), $p=0.12$ ]; in contrast, autonomic anxiety showed a positive correlation with CVC $[\beta(\mathrm{SE})=0.24(0.09), p$ $=0.01]$. In model IV, the total effect (direct plus indirect effect) of negative affectivity on CVC was not statistically significant $[\beta(\mathrm{SE})=-0.06(0.04), p=0.17]$. Furthermore, the indirect effects on CVC conferred by negative affectivity through anhedonic depression and autonomic anxiety were examined simultaneously in a multiple mediator model. The $\mathrm{BCa} 95 \% \mathrm{CI}$ showed that negative affectivity had significant but opposite indirect effects on CVC, either via anhedonic depression $[\beta(\mathrm{SE})=-0.05$
(0.03), 95\% CI: $-0.11,-0.01]$ or via autonomic anxiety $[\beta$ $(\mathrm{SE})=0.08$ (0.03), 95\% CI: 0.02, 0.15].

Figure 2 depicts how negative affectivity, under the hierarchical tripartite model, exerted its indirect effect on CVC. Panel A shows that the indirect effect of negative affectivity conferred by autonomic anxiety was conditioned (moderated) on negative affectivity itself. In general, increasing scores of negative affectivity tended to reverse this specific indirect effect from positive to negative. As such, higher negative affectivity reversed the relationship between autonomic anxiety and CVC. Once scores of negative affectivity reached $\geq 4$, the

Table 3 Multiple regression analysis on the association of cardiac vagal control and the Hospital Anxiety and Depression Scale $^{\dagger}$

\begin{tabular}{|c|c|c|}
\hline \multirow[b]{2}{*}{ Factor models } & \multicolumn{2}{|c|}{ High frequency component of heart rate variability $\left[\ln \left(m s^{2}\right)\right]$} \\
\hline & $\beta(\mathrm{SE})$ & P-value \\
\hline \multicolumn{3}{|l|}{ Model I. Unitary factor model of Razavi (1999) } \\
\hline Total score of full scale & $-0.01(0.01)$ & 0.46 \\
\hline \multicolumn{3}{|c|}{ Model II . Original two-factor model of Zigmond \& Snaith (1983) } \\
\hline Anhedonic depression* & $-0.21(0.09)$ & 0.02 \\
\hline Anxiety subscale & $0.02(0.04)$ & 0.54 \\
\hline \multicolumn{3}{|c|}{ Model III. Flat tripartite model of Dunbar et al. (2000) } \\
\hline Anhedonic depression & $-0.22(0.09)$ & 0.01 \\
\hline Negative affectivity & $-0.09(0.05)$ & 0.12 \\
\hline Autonomic anxiety & $0.24(0.09)$ & 0.01 \\
\hline \multicolumn{3}{|c|}{ Model IV. Hierarchical tripartite model of Dunbar et al. (2000) } \\
\hline \multicolumn{3}{|l|}{ Direct effect } \\
\hline Anhedonic depression & $-0.22(0.09)$ & 0.01 \\
\hline Negative affectivity & $-0.09(0.05)$ & 0.12 \\
\hline Autonomic anxiety & $0.24(0.09)$ & 0.01 \\
\hline Indirect effect & $\beta(\mathrm{SE})$ & BCa $95 \% \mathrm{Cl}^{\S}$ \\
\hline Negative affectivity $\rightarrow$ Anhedonic depression & $-0.05(0.03)$ & $(-0.11,-0.01)$ \\
\hline Negative affectivity $\rightarrow$ Autonomic anxiety & $0.08(0.03)$ & $(0.02,0.15)$ \\
\hline
\end{tabular}

†Variables forced to enter into the regression models include: Age (years), Education ( $\geqq 13,10-12,7-9,<7$ years), Diabetes mellitus (yes/no), Hypertension (yes/no), Cardiovascular disease (yes/no), Frequency of exercise ( $\geqq 1$ vs $<1 /$ week), Current smoker (yes/no), Site of data collection (Hospital vs home), Body mass index $\left(\geqq 25\right.$, vs $\left.<25 \mathrm{~kg} / \mathrm{m}^{2}\right)$, and various combination of factor items.

$\dagger$ Total score of depression subscale was rescaled by quartiles as $<5$ (lowest 2 quartiles), $5-6$ and $\geqq 7$.

$\S B C a$ : bias corrected and accelerated confidence interval derived from 5000 bootstrap samples. 

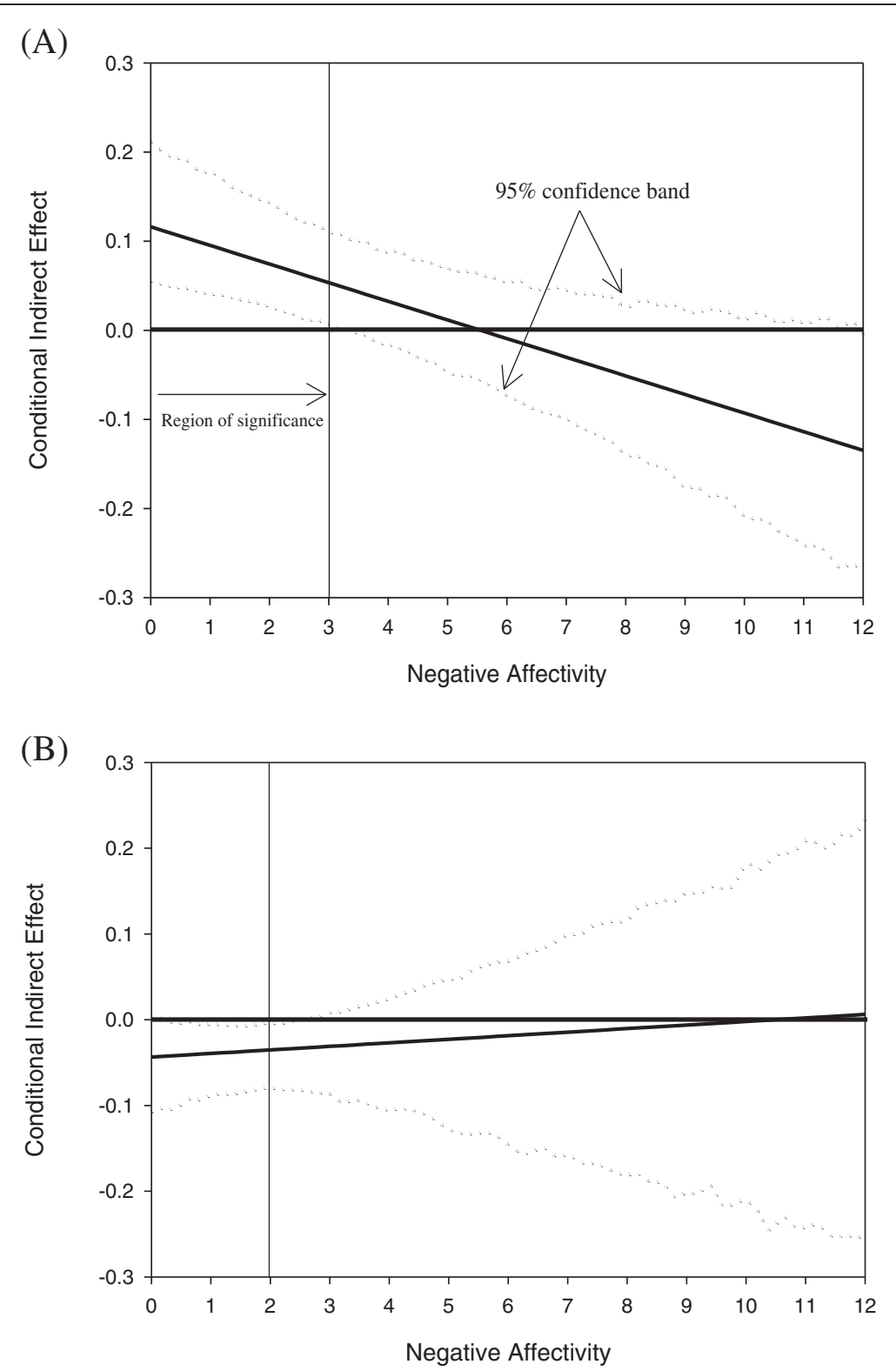

Figure 2 Plots of specific indirect effect on cardiac vagal control versus the moderator (negative affectivity). (A) conditional indirect effect mediated by autonomic anxiety (B) conditional indirect effect mediated by anhedonic depression.

specific indirect effect (i.e. negative affectivity $\rightarrow$ autonomic anxiety $\rightarrow$ CVC) became statistically non-significant. In parallel, panel B illustrates that the significant indirect effect of negative affectivity on CVC conferred by anhedonic depression (i.e. negative affectivity $\rightarrow$ anhedonic depression $\rightarrow$ CVC) was conditioned on scores of negative affectivity ranging from 0 to 3 . Therefore, as scores of negative affectivity reached 4 or more, the direct effects of anhedonic depression $[\beta$ (SE) $=-0.16(0.37), p=0.67]$ and autonomic anxiety $[\beta(\mathrm{SE})=-0.03(0.30), p=0.91]$ on CVC became non-significant but negative coefficients. The indirect effects that negative affectivity conferred through anhedonic depression and autonomic anxiety vanished as well, but the total effect of negative affectivity on CVC became statistically significant $[\beta(\mathrm{SE})=-0.41$ (0.19), $p=0.04]$.

\section{Discussion}

In the present study, a series of a priori-defined, theoretical factor solution of HADS were used to examine their psychometric conformity and psychophysiological relationship with CVC in elderly males residing in the community. Our results indicated that the flat three-factor model of HADS performed better in fitting the data but provided only a limited advantage over the hierarchical three-factor model and the original two-factor model. 
However, factors derived from the tripartite model offered an opportunity to scrutinize the counterbalancing effects of anhedonic depression and autonomic anxiety on CVC. In the hierarchical tripartite model, with lower negative affectivity, anhedonic depression and autonomic anxiety exerted counterbalancing effects on CVC along with corresponding indirect effects conferred by negative affectivity on CVC. In contrast, higher negative affectivity alone may reflect the overall deleterious effect of co-occurring anxiety and depression on CVC. Negative affectivity may be the unifying and potentially deleterious element linking individual trait negative emotions to impaired CVC [58]. The present study further illustrated how negative affectivity moderated the relationship between negative emotions and $\mathrm{CVC}$.

Theory-derived factor solutions have been previously utilized to investigate complex relationships between physiological indicators and co-occurring depression and anxiety. For example, the three hypothesized symptom groups in the tripartite model reflect highly distinctive patterns of brain activity [59-61]. CVC is another commonly applied psychophysiological indicator. The CVC has been of significant value in illuminating the process of basic dimensions of psychopathology and in predicting adverse health outcomes [62]. To the best of our knowledge, this is the first study that applies a series of a priori-defined, theory-based factor solutions to examine the relationship between $\mathrm{CVC}$ and co-occurring affective symptoms. Interestingly, our results suggest that clusters of affective symptoms derived from tripartite models as well as brain activity reflect distinctive patterns of CVC.

In terms of CVC, the results here seem to offer an advantage in utilizing the tri-dimensional scoring of HADS. This helps to disentangle the complex relationship between psychopathology and physiologic markers when applying the flat tripartite model in studies that explore the associations among $\mathrm{CVC}$ and co-occurring depression and anxiety. For example, by extracting symptoms of autonomic anxiety from the original anxiety subscale, we demonstrated that the absence of a correlation between the original HADS anxiety subscale and $\mathrm{CVC}$ resulted from the heterogeneous relationships between anxiety symptoms and CVC. From the perspective of the hierarchical arrangement of the three factors of the tripartite model, additional information is acquired as primacy is conferred on the factor of negative affectivity. In the present study, negative affectivity not only indirectly affected CVC via autonomic anxiety but also moderated the direct effect of autonomic affectivity on CVC. In other words, the higher order construct of affective symptoms may possess a determinant role in the way that lower order affective constructs affect CVC and eventually lead to adverse health outcomes.

According to the conceptual organization of the tripartite model, negative affectivity explains the covariation between depression and anxiety; however, depression and anxiety have distinct features not shared with each other or with negative affectivity [26]. With respect to cardiac autonomic control, the distinctive relationships between each tripartite factor of HADS and CVC, as noted in the present study, support this presumption. Paradoxically, the positive association between autonomic anxiety and CVC in the preset study subverts the typical understanding that elevated anxiety accompanies sympathetic activation and parasympathetic withdrawal. Several explanations help support this interesting finding.

First, the issues of construct and linguistic validity of autonomic anxiety in HADS may lead to the misallocation of symptom items and cause a biased association between autonomic anxiety and CVC. From the viewpoint of construct validity, Caci et al. (2003) argued that items assigned by Dunbar et al. (2000) were not suitable markers for the constructs of negative affectivity and autonomic anxiety. Furthermore, they were regarded as measuring the same thing [63]. However, in both the Chinese and non-Chinese literature examining the psychometric properties of HADS, the three items selected as markers of autonomic anxiety by Dunbar et al. (2000) nearly matched some forms of anxiety constructs, such as generalized anxiety, psychic anxiety, or panic [64]. Therefore, issues of item misallocation and language translation are insufficient to explain the positive correlation between autonomic anxiety and CVC as noted in this study. Despite arguments about inadequate psychometrical validity, the present study suggests that autonomic anxiety and negative affectivity as defined by Dunbar et al. (2000) seem to be different things in psychophysiological terms.

Another possible explanation is that Ahern et al. (2001) observed that inactivation of the right hemisphere by intracarotid sodium amobarbital administration resulted in a significant decrease in HRV. They suggested that the right hemisphere had a greater role in regulating cardiac function, perhaps by modifying parasympathetic effects [65]. Since autonomic anxiety is associated with the activation of the right parietal region [60], it is reasonable to infer that the positive association between autonomic anxiety and right hemisphere activation may contribute to increased CVC.

In the analysis of conditional indirect effect, we found that the positive contribution to $\mathrm{CVC}$ from autonomic anxiety was present only when scores of negative affectivity were low. The association tended to be reversed when negative affectivity scores were higher. 
Because negative affectivity in the tripartite model refers to "temperamental sensitivity to negative stimuli" and is related to neuroticism, low negative affectivity may represent less emotional arousal and a better coping style during stress. Constructive coping is related to better vagal tone [66]. Hence, in subjects with low negative affectivity, autonomic anxiety may merely be an external marker for good psychological flexibility in response to stress, which is associated with high vagal tone [67]. Several mild physical stresses have been reported to counteract the negative effects of aging and to increase longevity [68]. Herrmann et al. (2000) also reported the protective effect reflected in the HADS anxiety subscale among subjects who received routine exercise testing [69]. When subjects have high negative affectivity (a score of 4 for negative affectivity in this study), the combined effect on CVC from all factors of the tripartite model remains detrimental to health.

There are some limitations to the present study. First, although the flat tripartite model showed a better fit than did the hierarchical tripartite model and the original two-factor model, the difference was modest. Moreover, the goodness-of-fit indices of RMSEA and CFI suggested that none of these competitive models had a reasonably good fit. Empirically, the tripartite model of the European version of HADS provided a better and more acceptable fit to the data than did the twofactor model. In contrast, in the current study sample of elderly Chinese males, some elements may have affected the conformity of the tripartite model to the data. Structural differentiation of self-reported depression and anxiety is less easily performed in an older community sample than in younger populations [70]. In a Chinese population with coronary heart disease, Wang et al. (2006) also found structural ambiguity in the Chinese version of HADS [39]. They suggested that before any clear advantage of the tripartite models over the bidimensional models could be demonstrated, the amount of time consumed and the lack of a comparison to related literature rendered the tri-dimensional scoring approaches of HADS highly premature [39]. However, from the standpoint of CVC, the advantages of the analytic strategy of the tri-dimensional approach of HADS override the shortcoming of structural ambiguity. Because the present study did not aim to offer factor structures that conformed to the data with the most optimal goodness-of-fit, we preserved the original model specifications proposed by Dunbar et al. (2000); therefore, no model revisions were performed. Potential sources of specification errors are number of factors, indicators, and error theory. Further studies with larger sample sizes and indicators for latent variables should help to examine the conformity of the tripartite model among elderly Chinese. However, the development of psychometric measurements is usually driven by philology. Perhaps the validation of paper-and-pencil tests with biological markers, as in the present study, maximizes the practical utility of psychometric tools and goes beyond philology.

Second, the study subjects came from an urban community. Their scores for depression and anxiety were too low to generalize the study finding to a general clinical population. Subjects with higher negative affectivity were so few that the statistical power to detect a conditional indirect effect was compromised. However, the total effect of negative affectivity on CVC remained statistically significant when it was 4 or more. As such, the effect was strong enough to be detected even in a small sample. Nonetheless, further research with study subjects that include clinical patients are still necessary in order to determine whether negative affectivity actually moderates the association of autonomic anxiety with CVC.

Third, gender differences are noted in the link between depression and cardiovascular disease. The association between poorer CVC and depressive symptoms has been selectively observed in elderly males only of Chinese ethnicity [19]. However, other studies have demonstrated a female predominance in the link between depression and cardiovascular disease [5]. With respect to gender differences, whether or not different pathophysiological processes exist in different ethnic groups is unclear.

Finally, the confounding effect of medications could not be totally controlled in the present study. Because most classes of antidepressant have robust suppressive effects on CVC [71-75], subjects taking antidepressant were excluded in the present study. In addition to antidepressants, there are other medications with definite or probable effects on CVC, such as beta-blockers [76] and antipsychotics [77]. Unfortunately, we did not collect information on these medications, and future studies must assess these confounds.

\section{Conclusions}

In a comprehensive review, Rottenberg (2007) suggested the necessity of evaluating the effects of depression and anxiety on CVC, both independently and jointly [24]. The psychometric advantage of HADS echoes the requisites that Rottenberg (2007) called for. In the present study, individual associations between CVC and factors of theory-based models of HADS have been delineated among elderly males in the community. The composite scores of HADS have been dissected according to the tripartite model, and this provides the optimal application of HADS in identifying cardio-noxious affective symptoms. The significant and unique effects of negative affectivity and autonomic anxiety on CVC provide a more careful inspection of the way that anxiety symptoms confound the association between depression and 
CVC, not only in a co-occurring pattern but also in a heterogeneous form. The extent to which this strategy accurately predicts health outcomes remains unknown. Longitudinal studies are necessary to determine the clinical use of tri-dimensional HADS scoring, which is validated by virtue of CVC.

\section{Competing interests}

The authors declare that they have no competing interests.

\section{Authors' contributions}

HCC and PC analyzed the data and drafted the manuscript. CCHY and TBJK helped with the analysis and interpretation of HRV. TPS and PC participated in the study design and coordinated the conduct of the study. All authors read and approved the final manuscript.

\section{Author details}

'Department of Psychiatry and Center of Sleep Disorders, National Taiwan University Hospital, Taipei, Taiwan. ${ }^{2}$ Community Medicine Research Center \& Institute of Public Health, National Yang-Ming University, Shih-Pai, Taipei 112, Taiwan. ${ }^{3}$ Institution of Brain Science and Sleep Research Center, National Yang-Ming University, Taipei, Taiwan. ${ }^{4}$ Division of Psychiatry, National YangMing University, Taipei, Taiwan. ${ }^{5}$ Department of Psychiatry, Taipei Veterans General Hospital, Taipei, Taiwan.

Received: 16 July 2011 Accepted: 20 July 2012

Published: 30 July 2012

\section{References}

1. Aromaa A, Raitasalo R, Reunanen A, Impivaara O, Heliovaara M, Knekt P, Lehtinen V, Joukamaa M, Maatela J: Depression and cardiovascular diseases. Acta Psychiatr Scand 1994, 89(Suppl 377):77-82.

2. Wulsin $L R$, Singal $B M$ : Do depressive symptoms increase the risk for the onset of coronary disease? A systematic quantitative review. Psychosom Med 2003, 65:201-210.

3. Barefoot JC, Brummett BH, Helms MJ, Mark DB, Siegler IC, Williams RB: Depressive symptoms and survival of patients with coronary artery disease. Psychosom Med 2000, 62:790-795.

4. Pratt LA, Ford DE, Crum RM, Armenian HK, Gallo JJ, Eaton WW: Depression, psychotropic medication, and risk of myocardial infarction. Prospective data from the Baltimore ECA follow-up. Circulation 1996, 94:3123-3129.

5. Moller-Leimkuhler AM: Higher comorbidity of depression and cardiovascular disease in women: A biopsychosocial perspective. World J Biol Psychiatry 2010, 11:922-933.

6. Anda R, Williamson D, Jones D, Macera C, Eaker E, Glassman A, Marks J: Depressed affect, hopelessness, and the risk of ischemic heart disease in a cohort of U.S. adults. Epidemiology 1993, 4:285-294.

7. Schulz R, Beach SR, Ives DG, Martire LM, Ariyo AA, Kop WJ: Association between depression and mortality in older adults: the Cardiovascular Health Study. Arch Intern Med 2000, 160:1761-1768.

8. Kop WJ, Appels AP, de Leon CF M, de Swart HB, Bar FW: Vital exhaustion predicts new cardiac events after successful coronary angioplasty. Psychosom Med 1994, 56:281-287.

9. Irvine J, Basinski A, Baker B, Jandciu S, Paquette M, Cairns J, Connolly S, Roberts R, Gent M, Dorian P: Depression and risk of sudden cardiac death after acute myocardial infarction: testing for the confounding effects of fatigue. Psychosom Med 1999, 61:729-737.

10. Haines AP, Imeson JD, Meade TW: Phobic anxiety and ischaemic heart disease. Br Med J 1987, 295:297-299.

11. Kawachi I, Colditz GA, Ascherio A, Rimm EB, Giovannucci E, Stampfer MJ, Willett WC: Prospective study of phobic anxiety and risk of coronary heart disease in men. Circulation 1994, 89:1992-1997.

12. Herrmann C, Riedemann C, Bergmann G, Drinkmann A, Dumm A, Fritzsche K, Kanwischer H, Smeritschnig B, Stix P: Anxiety and depression in patients awaiting ICD implantation predict one-year incidence of malignant arrhythmias. Psychosom Med 1999, 61:114.

13. Berntson GG, Bigger JT Jr, Eckberg DL, Grossman P, Kaufmann PG, Malik M, Nagaraja HN, Porges SW, Saul JP, Stone PH, et al: Heart rate variability: origins, methods, and interpretive caveats. Psychophysiology 1997, 34:623-648.
14. Ter Horst GJ: Central autonomic control of the heart, angina, and pathogenic mechanisms of post-myocardial infarction depression. Eur $J$ Morphol 1999, 37:257-266.

15. Suess PE, Porges SW, Plude DJ: Cardiac vagal tone and sustained attention in school-age children. Psychophysiology 1994, 31:17-22.

16. George DT, Nutt DJ, Walker WV, Porges SW, Adinoff B, Linnoila M: Lactate and hyperventilation substantially attenuate vagal tone in normal volunteers. A possible mechanism of panic provocation? Arch Gen Psychiatry 1989, 46:153-156.

17. Musselman DL, Evans DL, Nemeroff CB: The relationship of depression to cardiovascular disease: epidemiology, biology, and treatment. Arch Gen Psychiatry 1998, 55:580-592.

18. Kawachi I, Sparrow D, Vokonas PS, Weiss ST: Decreased heart rate variability in men with phobic anxiety (data from the Normative Aging Study). Am J Cardiol 1995, 75:882-885.

19. Chen HC, Yang CCH, Kuo TBJ, Su TP, Chou P: Gender Differences in the Relationship between Depression and Cardiac Autonomic Function among Community Elderly. Int I Geriatr Psychiatry 2010, 25:314-322.

20. Thayer JF, Rossy LA, Ruiz-Padial E, Johnsen BH: Gender Differences in the Relationship between Emotional Regulation and Depressive Symptoms. Cognit Ther Res 2003, 27:349-364.

21. Gehi A, Mangano D, Pipkin S, Browner WS, Whooley MA: Depression and heart rate variability in patients with stable coronary heart disease: findings from the Heart and Soul Study. Arch Gen Psychiatry 2005, 62:661-666

22. de Jonge P, Mangano D, Whooley MA: Differential association of cognitive and somatic depressive symptoms with heart rate variability in patients with stable coronary heart disease: findings from the Heart and Soul Study. Psychosom Med 2007, 69:735-739.

23. Rottenberg J, Wilhelm FH, Gross JJ, Gotlib IH: Respiratory sinus arrhythmia as a predictor of outcome in major depressive disorder. J Affect Disord 2002, 71:265-272.

24. Rottenberg J: Cardiac vagal control in depression: a critical analysis. Biol Psychol 2007, 74:200-211.

25. Watson D, Clark LA, Carey G: Positive and negative affectivity and their relation to anxiety and depressive disorders. J Abnorm Psychol 1988, 97:346-353.

26. Clark LA, Watson D: Tripartite model of anxiety and depression: psychometric evidence and taxonomic implications. J Abnorm Psychol 1991, 100:316-336.

27. Watson D, Clark LA, Harkness AR: Structures of personality and their relevance to psychopathology. J Abnorm Psychol 1994, 103:18-31.

28. Zuckerman M, Kuhlman DM, Joireman J, Teta P: A comparison of three structural models for personality: The big three, the big five, and the alternative five. Pers Soc Psychol 1993, 65:757-768.

29. Watson D, Gamez W, Simms LJ: Basic dimensions of temperament and their relation to anxiety and depression: A symptom-based perspective. J Res Pers 2005, 39:46-66.

30. Watson D, Clark LA: Negative affectivity: the disposition to experience aversive emotional states. Psychol Bull 1984 96:465-490

31. Clark DA, Steer RA, Beck AT: Common and specific dimensions of self-reported anxiety and depression: implications for the cognitive and tripartite models. J Abnorm Psychol 1994, 103:645-654.

32. Steer RA, Clark DA, Beck AT, Ranieri WF: Common and specific dimensions of self-reported anxiety and depression: a replication. $J$ Abnorm Psychol 1995, 104:542-545.

33. Clark LA, Watson D, Mineka S: Temperament, personality, and the mood and anxiety disorders. J Abnorm Psychol 1994, 103:103-116.

34. Mineka S, Watson D, Clark LA: Comorbidity of anxiety and unipolar mood disorders. Annu Rev Psychol 1998, 49:377-412.

35. Watson D, Clark LA, Weber K, Assenheimer JS, Strauss ME, McCormick RA: Testing a tripartite model: II. Exploring the symptom structure of anxiety and depression in student, adult, and patient samples. J Abnorm Psychol 1995, 104:15-25.

36. Teachman BA, Siedlecki KL, Magee JC: Aging and symptoms of anxiety and depression: structural invariance of the tripartite model. Psychol Aging 2007, 22:160-170. 
37. Dunbar M, Ford G, Hunt K, Der G: A confirmatory factor analysis of the Hospital Anxiety and Depression scale: comparing empirically and theoretically derived structures. Br J Clin Psychol 2000, 39:79-94.

38. Martin CR, Thompson DR, Chan DS: An examination of the psychometric properties of the Hospital Anxiety and Depression Scale in Chinese patients with acute coronary syndrome. Psychiatry Res 2004, 129:279-288.

39. Wang W, Lopez V, Martin CR: Structural ambiguity of the Chinese version of the Hospital Anxiety and Depression Scale in patients with coronary heart disease. Health Qual Life Outcomes 2006, 4:6.

40. Clark LA, Watson D, Leeka J: Diurnal variation in the positive affects. Motiv Emot 1989, 13:205-234.

41. Nitschke JB, Heller W, Palmieri PA, Miller GA: Contrasting patterns of brain activity in anxious apprehension and anxious arousal. Psychophysiology 1999, 36:628-637.

42. Wardenaar KJ, Vreeburg SA, van Veen T, Giltay EJ, Veen G, Penninx BW, Zitman FG: Dimensions of depression and anxiety and the hypothalamo-pituitary-adrenal axis. Biol Psychiatry 2011, 69:366-373.

43. Veen $\mathrm{G}$, van Vliet IM, DeRijk RH, Giltay EJ, van Pelt J, Zitman FG: Basal cortisol levels in relation to dimensions and DSM-IV categories of depression and anxiety. Psychiatry Res 2011, 185:121-128.

44. Bjelland I, Dahl AA, Haug T, Neckelmann D: The validity of the Hospital Anxiety and Depression Scale. An updated literature review. J Psychosom Res 2002, 52:69-77.

45. Leung CM, Ho S, Kan CS, Hung CH, Chen CN: Evaluation of the Chinese version of the Hospital Anxiety and Depression Scale. A cross-cultural perspective. Int J Psychosom 1993, 40:29-34.

46. Razavi D, Delvaux N, Farvacques C, Robaye E: Screening for adjustment disorders and major depressive disorders in cancer in-patients. $\mathrm{Br} J$ Psychiatry 1990, 156:79-83.

47. Zigmond AS, Snaith RP: The hospital anxiety and depression scale. Acta Psychiatr Scand 1983, 67:361-370

48. Kuo TBJ, Chan SHH: Continuous, on-line, real-time spectral analysis of systemic arterial pressure signals. Am J Physiol 1993, 264:H2208-H2213.

49. Lam CL, Pan PC, Chan AW, Chan SY, Munro C: Can the Hospital Anxiety and Depression (HAD) Scale be used on Chinese elderly in general practice? Fam Pract 1995, 12:149-154.

50. Hayes AF: Beyond Baron and Kenny: Statistical Mediation Analysis in the New Millennium. Commun Monogr 2009, 76:408-420.

51. Preacher KJ, Hayes AF: Asymptotic and resampling strategies for assessing and comparing indirect effects in multiple mediator models. Behav Res Methods 2008, 40:879-891.

52. Baron RM, Kenny DA: The moderator-mediator variable distinction in social psychological research: conceptual, strategic, and statistical considerations. J Pers Soc Psychol 1986, 51:1173-1182.

53. Sobel ME: Asymptotic confidence intervals for indirect effects in structural equation models. In Sociological methodology. Edited by Leinhardt S. San Francisco: Jossey-Bass; 1982:290-212.

54. Sobel ME: Some new results on indirect effects and their standard errors in covariance structure models. In Sociological methodology. Edited by Tuma N. Washington, DC: American Sociological Association; 1986:159-186.

55. Mackinnon DP, Lockwood CM, Williams J: Confidence Limits for the Indirect Effect: Distribution of the Product and Resampling Methods. Multivariate Behav Res 2004, 39:99.

56. Williams J, Mackinnon DP: Resampling and Distribution of the Product Methods for Testing Indirect Effects in Complex Models. Struct Equ Modeling 2008, 15:23-51.

57. Preacher KJ, Rucker DD, Hayes AF: Addressing Moderated Mediation Hypotheses: Theory, Methods, and Prescriptions. Multivariate Behav Res 2007, 42:185-227.

58. Bleil ME, Gianaros PJ, Jennings JR, Flory JD, Manuck SB: Trait negative affect: toward an integrated model of understanding psychological risk for impairment in cardiac autonomic function. Psychosom Med 2008 70:328-337.

59. Bruder GE, Fong R, Tenke CE, Leite P, Towey JP, Stewart JE, McGrath PJ, Quitkin FM: Regional brain asymmetries in major depression with or without an anxiety disorder: a quantitative electroencephalographic study. Biol Psychiatry 1997, 41:939-948.

60. Heller W, Etienne MA, Miller GA: Patterns of perceptual asymmetry in depression and anxiety: implications for neuropsychological models of emotion and psychopathology. J Abnorm Psychol 1995, 104:327-333.
61. Tomarken AJ, Keener AD: Frontal brain asymmetry and depression: A self-regulatory perspective. Cogn Emot 1998, 12:387-420.

62. Friedman $\mathrm{BH}$ : An autonomic flexibility-neurovisceral integration model of anxiety and cardiac vagal tone. Biol Psychol 2007, 74:185-199.

63. Caci H, Bayle FJ, Mattei V, Dossios C, Robert P, Boyer P: How does the Hospital and Anxiety and Depression Scale measure anxiety and depression in healthy subjects? Psychiatry Res 2003, 118:89-99.

64. Leung CM, Wing YK, Kwong PK, Lo A, Shum K: Validation of the Chinese-Cantonese version of the hospital anxiety and depression scale and comparison with the Hamilton Rating Scale of Depression. Acta Psychiatr Scand 1999, 100:456-461.

65. Ahern GL, Sollers JJ, Lane RD, Labiner DM, Herring AM, Weinand ME, Hutzler R, Thayer JF: Heart rate and heart rate variability changes in the intracarotid sodium amobarbital test. Epilepsia 2001, 42:912-921.

66. Fabes RA, Eisenberg N: Regulatory control and adults' stress-related responses to daily life events. J Pers Soc Psychol 1997, 73:1107-1117.

67. Appelhans BM, Luecken LJ: Heart Rate Variability as an Index of Regulated Emotional Responding. Rev Gen Psychology 2006, 10:229-240.

68. Minois N: Longevity and aging: beneficial effects of exposure to mild stress. Biogerontology 2000, 1:15-29.

69. Herrmann C, Brand-Driehorst S, Buss U, Ruger U: Effects of anxiety and depression on 5-year mortality in 5,057 patients referred for exercise testing. J Psychosom Res 2000, 48:455-462.

70. Meeks S, Woodruff-Borden J, Depp CA: Structural differentiation of self-reported depression and anxiety in late life. J Anxiety Disord 2003, 17:627-646.

71. Rechlin T, Weis M, Claus D, Kaschka WP: Identifying delirious states and autonomic cardiovascular dysfunction associated with amitriptyline treatment by standardized analysis of heart rate. Psychiatry Res 1995 56:279-287.

72. Tulen JH, Bruijn JA, de Man KJ, Pepplinkhuizen L, van den Meiracker AH, Man in 't Veld AJ: Cardiovascular variability in major depressive disorder and effects of imipramine or mirtazapine (Org 3770). J Clin Psychopharmacol 1996, 16:135-145.

73. Agelink MW, Majewski T, Wurthmann C, Postert T, Linka T, Rotterdam S, Klieser E: Autonomic neurocardiac function in patients with major depression and effects of antidepressive treatment with nefazodone. $J$ Affect Disord 2001, 62:187-198.

74. Bar KJ, Greiner W, Jochum T, Friedrich M, Wagner G, Sauer H: The influence of major depression and its treatment on heart rate variability and pupillary light reflex parameters. J Affect Disord 2004, 82:245-252.

75. Licht CM, de Geus EJ, Zitman FG, Hoogendijk WJ, van Dyck R, Penninx BW: Association between major depressive disorder and heart rate variability in the Netherlands Study of Depression and Anxiety (NESDA). Arch Gen Psychiatry 2008, 65:1358-1367.

76. Lampert R, Ickovics JR, Viscoli CJ, Horwitz RI, Lee FA: Effects of propranolol on recovery of heart rate variability following acute myocardial infarction and relation to outcome in the Beta-Blocker Heart Attack Trial. Am J Cardiol 2003, 91:137-142.

77. Silke B, Campbell C, King DJ: The potential cardiotoxicity of antipsychotic drugs as assessed by heart rate variability. J Psychopharmacol 2002, $16: 355-360$

doi:10.1186/1471-244X-12-93

Cite this article as: Chen et al:: Cardiac vagal control and theoretical models of co-occurring depression and anxiety: A

cross-sectional psychophysiological study of community elderly. BMC Psychiatry 2012 12:93. 